\title{
Impacto antrópico en el Manto Acuífero Barva (Heredia, Costa Rica) con énfasis en el uso del suelo (1992-2006)
}

\author{
Gustavo Castro Peinador ${ }^{1}$, Francisco José Chavarría Ñamendi ${ }^{2}$, Juan Camilo de la Cruz Godoy ${ }^{3}$, Cecilia Gelabert ${ }^{4}$, \\ David Martínez Cascante, Wendy Carolina Paniagua Palacios, Natalie Viviana Sánchez Ulate, Karen Daniela Sibaja \\ Morales \& Arianna Gisela Tejeda Tellez \\ Instituto Internacional en Conservación y Manejo de Vida Silvestre, Universidad Nacional. Apdo. 1350-3000. Heredia, Costa Rica. \\ 'yaguarcr@yahoo.com; ${ }^{2}$ chava76@gmail.com; ${ }^{3}$ juandelacruzgo@gmail.com; ${ }^{4}$ Autora de correspondencia: gelabert@agro.uba.ar
}

\begin{abstract}
Anthropic impact of the Barva aquifer (Heredia, Costa Rica) with emphasis on land use (1992-2006). The advance of urbanization in the Central Valley of Costa Rica is having negative impacts on land use and stresses the environment with a high demand of natural resources, particularly water resources. One example is the Barva Water Table, one of the main water suppliers of semi-urban areas of the Greater Metropolitan Area of Costa Rica, which leads to conflicts between social actors involved with this resource. Comparing land use maps of the area defined by Act LXV of 1888, as well as the literature, we found that forest cover is decreasing while area covered by crops and pastures has increased from 1992 and 2006. At the same time, the forest area has undergone a process of fragmentation, and the increasing number of forest patches has an edge effect in all classes of land use. We recommend sustainable governance of water resources in the protected area's buffer zone, with the participation of the local government, development associations, and other related public and private institutions.
\end{abstract}

\section{KEY WORDS}

Aquifer, Costa Rica, fragmentation, land use.

\section{RESUMEN}

El gran avance de la urbanización en el Valle Central de Costa Rica está generando impactos negativos sobre el uso del suelo y está sometiendo al ambiente a intensas presiones debido a la gran demanda de los recursos naturales, particularmente del hídrico. El Manto Acuífero Barva, una de las principales fuentes de agua que abastece zonas urbanas y semiurbanas de la Gran Área Metropolitana, actualmente se encuentra bajo una fuerte presión de cambio de uso en el suelo, lo que a su vez genera conflictos entre diferentes actores sociales involucrados con este recurso. Comparando los mapas de uso de suelo de 1992 y 2006 referentes a la zona delimitada por la Ley LXV de 1888, así como diversas fuentes bibliográficas, se observa que el área boscosa está disminuyendo mientras que la zona de cultivos y pasturas ha aumentado. Asimismo, la región ha sufrido un proceso de fragmentación, lo que se refleja en el aumento de parches, así como del efecto de borde en todas las clases de uso de suelo. Se recomienda iniciar un proceso de gestión participativa y de gobernabilidad sostenible del recurso hídrico en la zona de amortiguamiento del área protegida y así desarrollar acciones de manera conjunta con las comunidades ubicadas en la zona de influencia, con los gobiernos locales, asociaciones de desarrollo, instituciones públicas y privadas interesadas o directamente relacionadas con la protección, conservación y manejo sostenible de los recursos hídricos.

PALABRAS CLAVE

Acuífero Barva, Costa Rica, fragmentación, uso de suelo. 
En las últimas décadas, el área urbana del Valle Central de Costa Rica ha avanzado sobre las zonas periurbanas generando grandes impactos negativos, conformando un territorio inestable en cuanto a los diferentes usos de la tierra y sometiendo al ambiente a intensas presiones (Alvarado 2003), debido a la fuerte demanda de los recursos naturales, particularmente sobre los hídricos, los cuales se ven afectados también por contaminación superficial y subterránea (Ureña 2004). Por esta razón, la ubicación de las zonas de recarga acuífera y la comprensión de la dinámica de los procesos hidrológicos es de vital importancia para evaluar el riesgo de contaminación de las aguas subterráneas y para definir zonas de protección de los acuíferos (Reynolds-Vargas \& Fraile-Merino 2002).

Las fuentes de aguas subterráneas constituyen el principal abastecimiento de agua potable de manera natural y a su vez representan un ahorro de energía y recursos económicos debido a que no necesitan de bombeo para su aprovechamiento (Vargas 2002). Sin embargo, para un adecuado manejo de estas zonas y de las de recarga de los acuíferos (como un primer paso para la protección), es necesario llevar a cabo un inventario de los manantiales utilizados y del caudal de explotación promedio.

En Costa Rica, la Ley de Agua de 1942, que aún está vigente, en su Artículo 31 declara como reserva a favor de la Nación las tierras que circunden los sitios de captación o tomas surtidoras de agua potable en el área delimitada por una circunferencia cuyo radio posea un valor mínimo de $200 \mathrm{~m}$ y también a la zona forestal que protege el conjunto de terrenos en la que se produce la infiltración de agua. Dicha Ley, en su Artículo 149 establece la prohibición de destruir los árboles situados a menos de $70 \mathrm{~m}$ de los manantiales que nazcan en cerros o a menos de $50 \mathrm{~m}$ de los que nazcan en terrenos planos. Asimismo, en el Artículo 33, señala como área de protección aquellas que bordean nacientes permanentes (Vargas 2002). Sin embargo, se desconoce cómo han sido determinadas estas distancias y si las mismas son efectivas en términos de captación de agua.

En los últimos años, el avance de la urbanización sin ningún tipo de planificación ha impactado en el paisaje de forma directa por medio de la fragmentación de las áreas naturales e indirecta dado que compiten con la producción agropecuaria por el recurso tierra, lo que genera una extensión de la frontera agrícola hacia las áreas naturales (Alvarado 2003).

El crecimiento urbano en la región delimitada por la Ley LXV de 1888 representa uno de los mayores impactos antrópicos al ciclo hidrológico, debido a la reducción en la permeabilidad de los suelos por el desarrollo de infraestructura que aísla totalmente la capa edáfica. De esta forma, el asfalto y el concreto reemplazan al suelo, provocando efectos posteriores como inundaciones y deslizamientos. Otro impacto de la urbanización sobre el recurso hídrico es la producción y tratamiento de aguas negras, ya que generan contaminación (Vargas 2002). Estas condiciones se agudizan cuando el proceso de ocupación residencial es disperso y las municipalidades no realizan un monitoreo detallado de las características de operación de las residencias, especialmente durante las etapas de construcción (Ramírez \& Alfaro 2002, Morera et al. 2007).

El uso agrícola también disminuye la permeabilidad de los suelos y aumenta la escorrentía superficial. En el caso de los espacios del periurbano rural, la preocupación se centra en el alto nivel de plaguicidas y con ello las posibilidades de contaminación, debido al exceso en su uso en algunas nuevas actividades agrícolas como las plantas ornamentales, al tiempo que la infraestructura recubierta por sarán negro contrasta y desvaloriza el paisaje de montaña (Ramírez \& Alfaro 2002, Morera et al. 2007).

El Acuífero Barva (localizado en las provincias de Heredia, San José y Alajuela al suroeste del Parque Nacional Braulio Carrillo, PNBC) es uno de los principales acuíferos que abastecen áreas urbanas y semiurbanas de la Gran Área Metropolitana (GAM) (Ureña 2004). Por tal motivo, desde hace más de un siglo existe preocupación por las autoridades costarricenses en procurar su protección, hecho que se hace manifiesto en el Decreto Ley No. LXV del 30 de julio de 1888, en el cual se delimita una zona de protección que abarca los cantones Heredia, Santa Bárbara, Barva, San Rafael, San Isidro (todos estos en Heredia) y Vázquez de Coronado (en San José).

El presente informe se basa en la revisión bibliográfica de documentos oficiales y en la elaboración de mapas de la zona en cuestión a partir de capas de uso de la tierra, con el fin de organizar y sintetizar la información existente para así, brindar recomendaciones de acción a corto y mediano plazo para un manejo adecuado del área delimitada por la Ley LXV.

\section{METODOLOGÍA}

\section{Área de estudio}

El área bajo estudio se ubica en las provincias de Heredia y San José, en los cantones de Barva, San Rafael, San Isidro, Santa Bárbara, Heredia y Vázquez de Coronado. Incluye los Refugios de Vida Silvestre Cerro Dantas y Jaguarundi y colinda al norte con el Parque Nacional Braulio Carrillo (Fig. 1). 

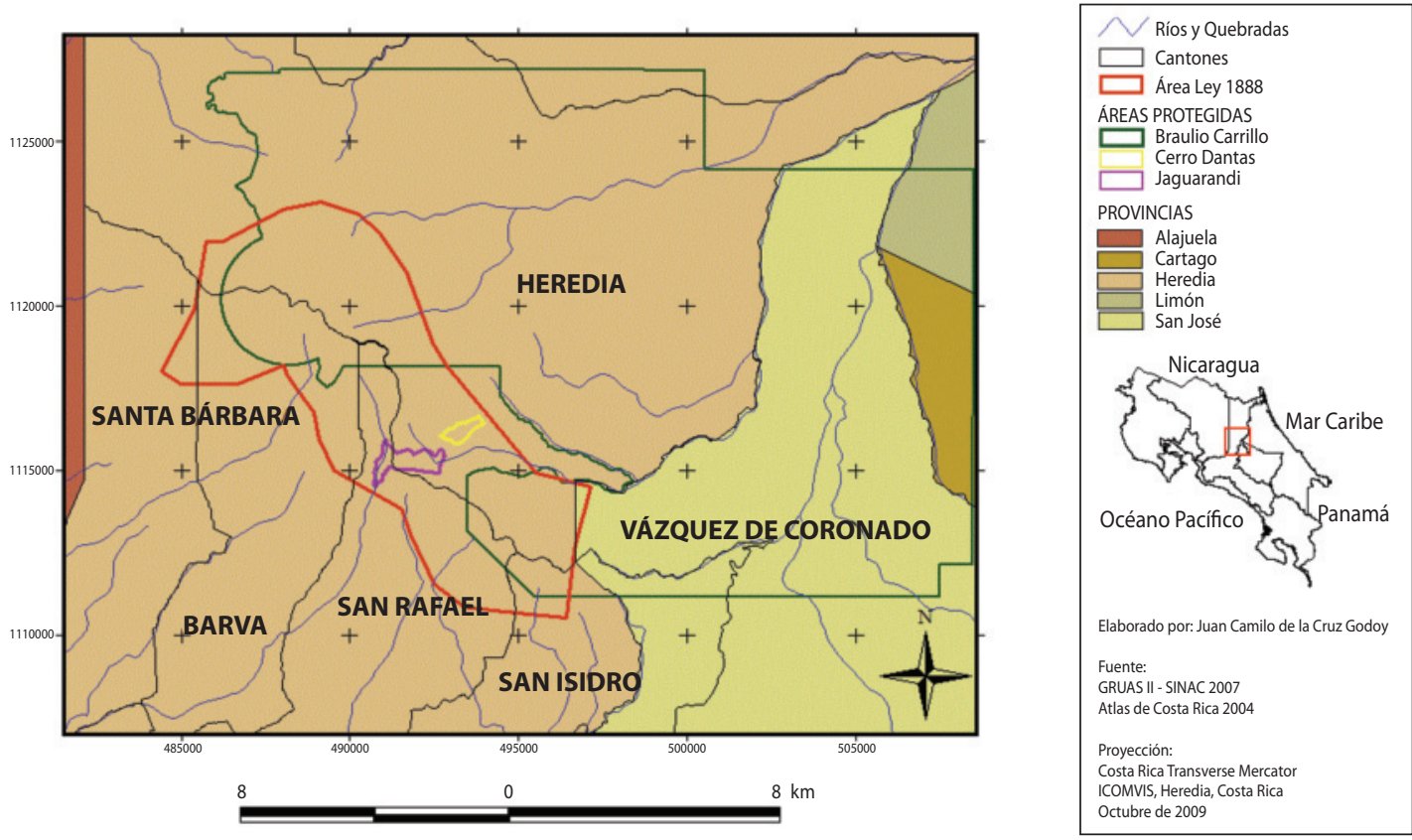

FIG. 1. Localización del área delimitada por el Decreto Ley No. LXV del 30 de julio de 1888.

\section{Recopilación y análisis bibliográfico}

Se realizó una revisión exhaustiva de los documentos existentes sobre la evolución y el estado actual del uso del suelo en la región delimitada por la Ley LXV de 1888 y sus zonas aledañas con el fin de ordenar y comparar la información que estos presentan.

\section{Análisis de la información geográfica}

Mediante el uso de ArcView 3.3 (Environmental Systems Research Institute 2002) se digitalizó el área de estudio según la hoja cartográfica Barva, provista por José Francisco Alfaro, la cual tenía el polígono con el área protegida por el Decreto Ley No. LXV de 1888. A partir de dicho polígono, se identificó dos áreas de amortiguamiento utilizando la herramienta XTOOLS. La primera de ellas bordea el área protegida como un cinturón de $1 \mathrm{~km}$ de ancho, mientras que la segunda, de igual ancho, rodea el perímetro externo de la primer área de amortiguamiento.

La caracterización del uso del suelo del área bajo estudio se realizó sobre la base de los archivos de "Uso del suelo 1992" (Ministerio de Agricultura y Ganadería, resolución $30 \times 30 \mathrm{~m}$ ) y de los archivos generados por el proyecto GRUAS II (Sistema Nacional de Áreas de Conservación 2007). Posteriormente, se reclasificó la caracterización de uso del suelo del área delimitada por la Ley LXV en cuatro categorías: a) Bosque, b) Cultivos, c) Charral y d) Pasto. Debido a que el archivo del 2006 contenía zonas sin datos, se procedió a borrar las mismas en el archivo de 1992 para homogeneizar el resultado de los análisis. El paso anterior sobreestimó el número de parches y bordes de los dos archivos, por lo que una vez corrido el análisis de métricas de paisaje (con la ayuda de la extensión PatchAnalysis 3.0) se procedió a restar las métricas del 2006 a las de 1992 para anular esta alteración de los archivos y a utilizar los datos de la diferencia entre los periodos para interpretar los resultados.

\section{RESULTADOS}

\section{Recarga del Acuífero Barva}

La recarga de este acuífero tiene su origen en las precipitaciones que se presentan en las partes altas de la cuenca del río Virilla. Dicha zona cumple una función de recarga de acuíferos profundos por medio de infiltración en los lechos de los ríos. Algunos de estos manantiales son utilizados para abastecer acueductos administrados por ESPH y AyA (Vargas 2002). A partir de estas zonas de infiltración, es posible definir áreas de protección y conservación, por lo que toda actividad potencialmente 
contaminante debe ser controlada o limitada a un nivel adecuado dentro de la zona para evitar amenazas de contaminación de agua (Vargas 2002). Sin embargo, debido a cuestiones socioeconómicas, estas limitaciones resultan difíciles de cumplir en su sentido amplio, ya que en la práctica se opta por realizar una división de las áreas de captación en zonas menores (las más cercanas a los acuíferos) para aplicar restricciones más severas llamándolas áreas de protección máxima (Alvarado 2003).

Este proceso de cambio de uso del suelo, ha generado conflictos entre los principales actores del área de estudio, dando lugar a gran cantidad de procesos legales y recursos de amparo que han sido aceptados por la Sala IV Constitucional con el fin de proteger la zona de recarga acuífera (Apéndice 1 y 2).

\section{Paisaje natural}

Las zonas que protege el Decreto Ley LXV de 1888 poseen un gradiente altitudinal en el que se identifican cuatro zonas de vida: a) bosque muy húmedo montano bajo, b) bosque pluvial montano, c) bosque pluvial montano bajo y d) bosque pluvial montano transición a montano bajo; así como una variedad de especies vegetales y animales típicos de los bosques montanos (Bolaños \& Watson 1993).

Las precipitaciones medias anuales son de $2400 \mathrm{~mm}$, prevaleciendo un promedio de temperatura de $21^{\circ} \mathrm{C}$. La estación seca va desde diciembre a abril y la estación lluviosa comprende de mayo a noviembre (Ramírez 2005). Los suelos predominantes son los andisoles con alta pedregosidad y con pH ácido entre 5,1 a 5,8 (Baraona \& Rivera 1995).

Gran parte del área protegida por la Ley LXV de 1888 se ubica dentro del Parque Nacional Braulio Carrillo, donde se destaca el volcán Barva, con 2906 msnm, que posee un bosque maduro con vegetación siempre verde de gran densidad y complejidad florística entre las que predominan varios géneros: Ardisia, Brunellia, Clethra, Dendropanax, Drimys, llex, Schefflera, Viburnum y Weinmannia, y algunas especies de las familias Lauraceae y Myrsinaceae (Ramírez 2005).

Conocer las características de sus hábitats y los procesos de fragmentación a nivel de paisaje son insumos esenciales para la elaboración de estrategias de conservación. Además, el conocer y valorar la importancia ecológica de las especies animales y vegetales y sus relaciones con los diferentes ecosistemas presentes en el sector que protege la Ley de 1888 , dará pautas importantes para futuras prácticas de manejo tendientes a proteger el conjunto del ecosistema que rodea este Parque y principalmente la recarga hídrica que posee dicha zona (Ramírez 2005).
En la actualidad, la zona de recarga acuífera Barva y alrededores está sometida a una gran presión de cambio de uso del suelo como consecuencia de la transformación de áreas de bosques, pastos y café a sistemas urbanos, comerciales e industriales. El paisaje rural y agropecuario en esta zona ha estado sometido a políticas económicas, liberalización financiera y apertura comercial que ha creado oportunidades para sectores vinculados al mercado de tierras, al turismo, al desarrollo urbano y residencial de altas inversiones, así como el desarrollo de cultivos no tradicionales (viveros ornamentales) (Comisión Interinstitucional de Micro cuencas de Heredia 2005).

El crecimiento acelerado de la población que habita en la GAM augura un incremento en la demanda de agua para uso humano, por lo cual es fundamental tomar decisiones que permitan rescatar las áreas de recarga acuífera que podrían ser utilizadas para este fin. En la actualidad, un alto porcentaje del agua superficial utilizada en el área metropolitana proviene del Embalse El Llano y el río Tiribí (Reynolds-Vargas \& Fraile-Merino 2002). Sin embargo, a futuro estas fuentes no darán abasto.

\section{Caracterización de uso del suelo}

Se han obtenido dos mapas de uso del suelo de dos períodos diferentes: 1992 (Fig. 2) y 2006 (Fig. 3) con el fin de comparar el cambio de cobertura en el área bajo estudio. Cabe destacar que los archivos de usos del paisaje utilizados para este análisis fueron digitalizados mediante diferentes métodos, por consiguiente puede existir algún sesgo en los resultados obtenidos en este estudio, propiciando que haya un mayor número de parches en el mapa que fue digitalizado de forma automatizada (mapa año 2006) con respecto al que fue digitalizado de forma manual (mapa año 1992).

Según la diferencia en el área total por clase en el uso del suelo entre 1992 y 2006 (Fig. 4) se manifiesta el mismo patrón en todas las áreas analizadas: un descenso en el área boscosa mientras que la destinada a cultivos y pastos incrementó, así como el charral. Esta tendencia era esperada para las zonas de amortiguamiento; sin embargo, es notable que la zona protegida haya perdido más de 1000 ha de bosque natural durante el periodo analizado. Lo anterior contrasta con la tendencia general de la GAM, donde la cobertura boscosa aumentó (Morera et al. 2007). Se destaca que el estudio de Morera y colaboradores (2007) consideró el charral, bosque denso y poco denso junto con las plantaciones forestales como cobertura boscosa y en este estudio se utilizaron categorías diferentes. También se debe considerar que el presente trabajó utiliza un área de estudio más pequeña. 
Mapa de uso del suelo 1992

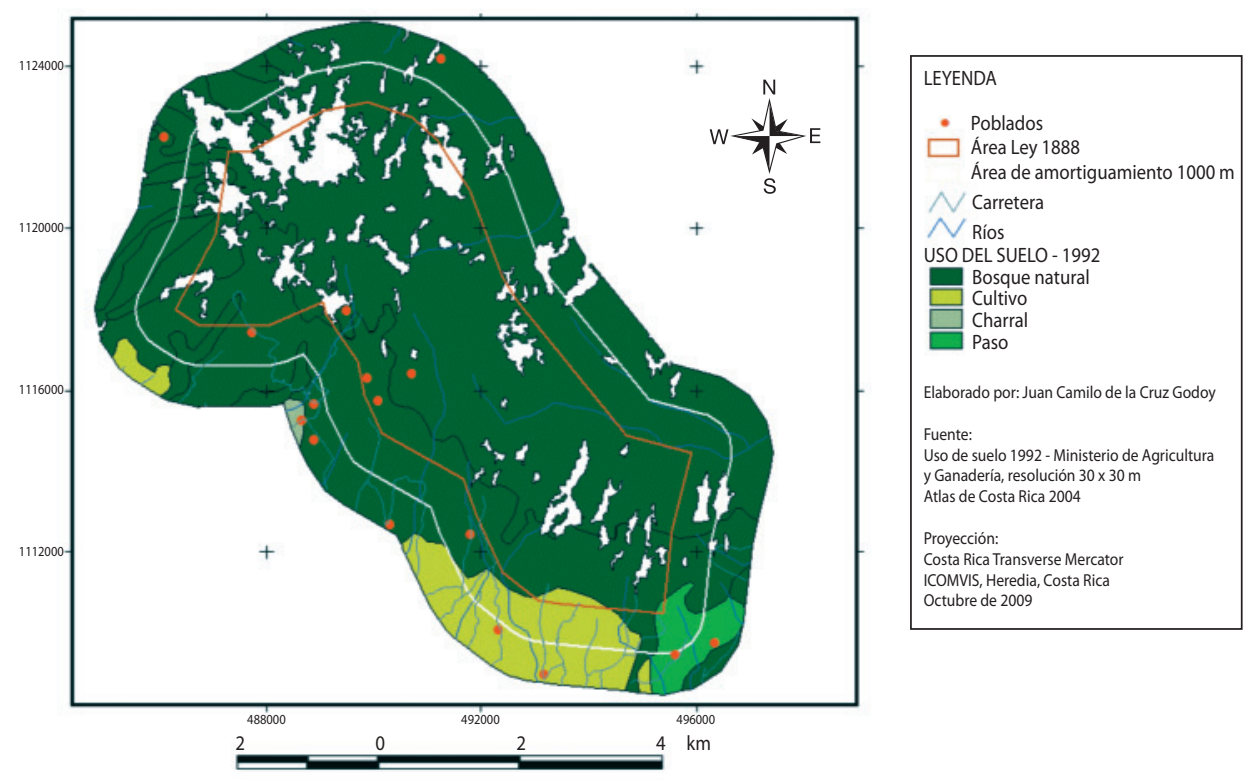

FIG. 2. Cobertura vegetal de la zona delimitada por la Ley LXV de 1888, basada en el mapa de uso de suelo de 1992.

Mapa de uso del suelo 2006

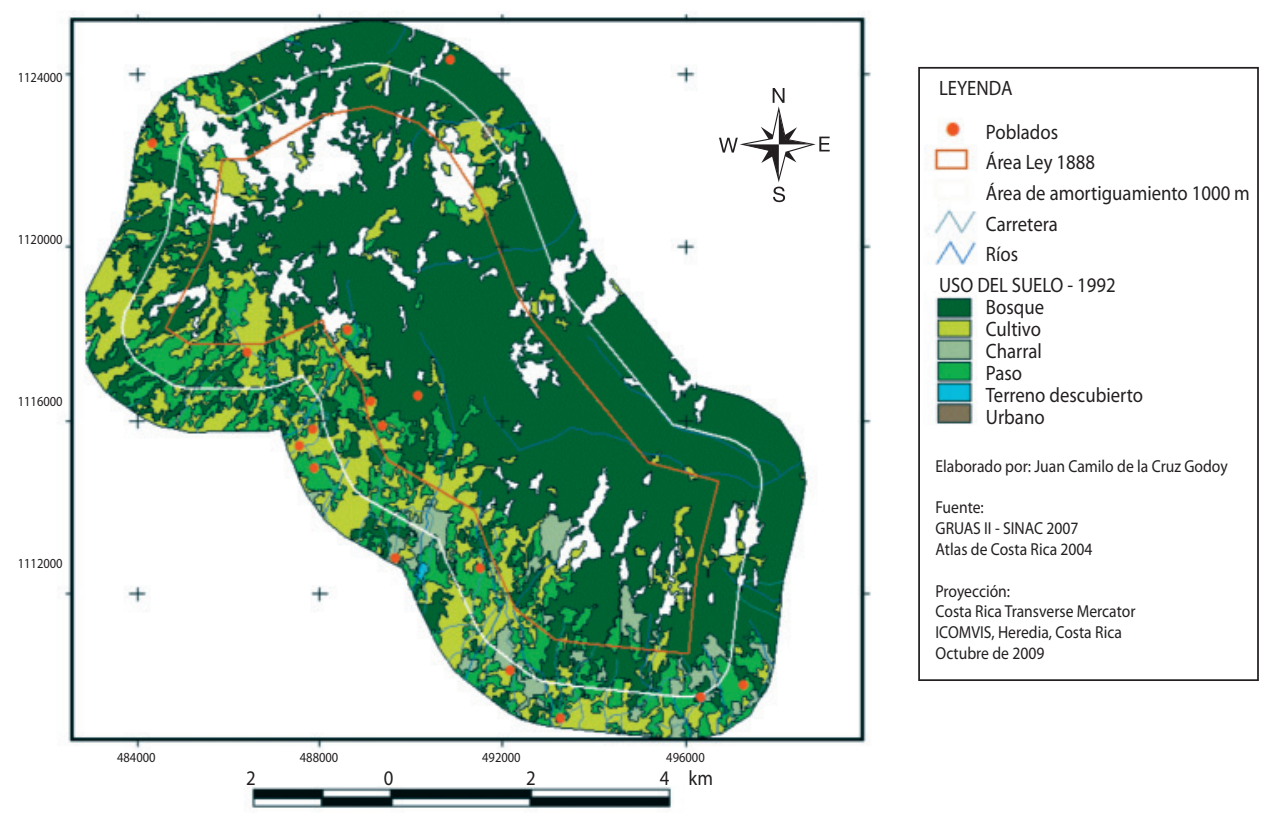

FIG. 3. Uso de suelo de la zona delimitada por la Ley LXV de 1888, basado en el mapa de uso de suelo del 2006. 


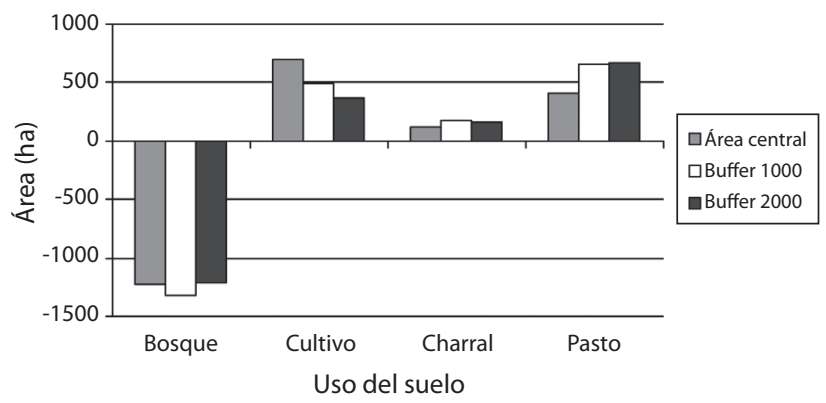

FIG. 4. Diferencia del área total por clase de uso de suelo en la zona de estudio, en el área de amortiguamiento de $1 \mathrm{~km}$ y en el área de amortiguamiento de $2 \mathrm{~km}$.

El número de parches ha incrementado en todas las clases de uso de suelo. Para el bosque, esto significa que la fragmentación ha aumentado y, potencialmente, se ha perdido conectividad. Al tener en cuenta que el área de cultivos disminuyó y que el número de fragmentos aumentó (Fig. 5), una posible explicación es que se cambió la práctica de cultivos extensivos por cultivos intensivos (Fig. 2 y 3). La explicación anterior también aplica para el cambio en los pastos. Mientras tanto, los resultados para el charral en la zona central y en la de amortiguamiento de $1000 \mathrm{~m}$ indican un incremento en el número de parches, ya que el aumento promedio del tamaño de estos, junto con el aumento del número de parches de charral no se generaron por la división de fragmentos más grandes sino por la generación de nuevos parches (Fig. 6).

Aún cuando las prácticas de cultivo y ganadería cambiaron de extensivas a pequeñas fincas, el área sumada de todas estas fincas incrementó el área de estos usos del suelo. Lo anterior indica una fuerte presión en los bosques de la zona para convertirlos en tierras comercialmente productivas.

Otro indicador de fragmentación es el aumento de borde total en todas las clases (Fig. 7). El borde se incrementa conforme un parche se vuelve más irregular y la relación perímetro-área crece es decir: los parches de cada clase se hacen más pequeños en área y más complejos en forma. Para la vida silvestre, el incremento en el efecto de borde puede traer consecuencias negativas, por ejemplo, la intrusión de especies competidoras dentro de su hábitat o el cambio del microclima al que están acostumbrados, debido a la reducción del amortiguamiento que da el borde a la zona núcleo del parche de hábitat.

El aumento en el borde promedio de la zona de recarga acuífera significa que ésta se ha vuelto más compleja y que

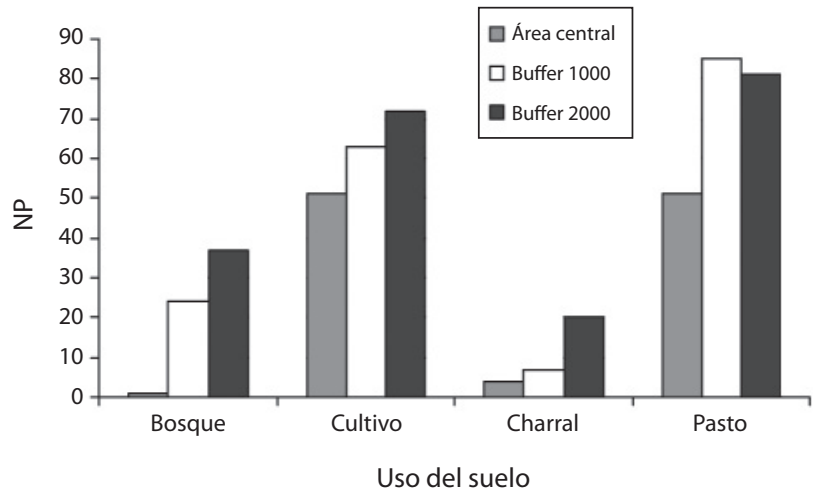

FIG. 5. Diferencia en el número de parches por clase de uso de suelo en la zona de estudio, en el área de amortiguamiento de $1 \mathrm{~km}$ y en el área de amortiguamiento de $2 \mathrm{~km}$.

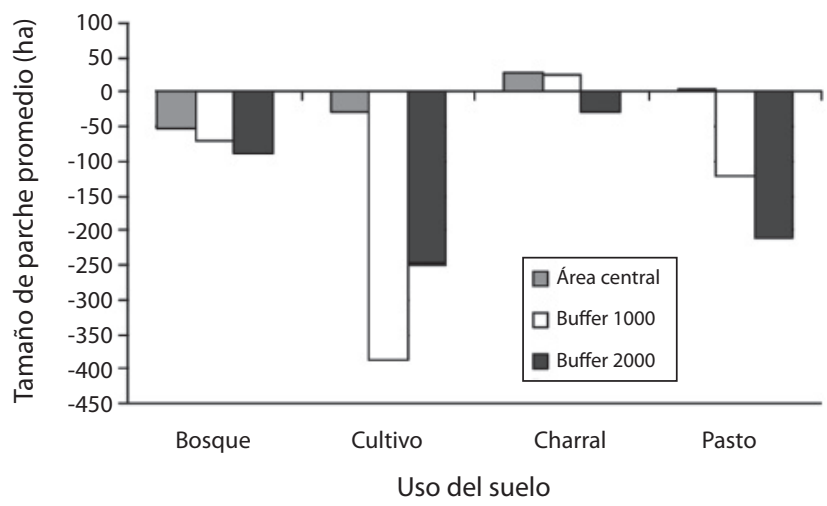

FIG. 6. Diferencia en el promedio del tamaño del parche por clase de uso de suelo en la zona de estudio, en el área de amortiguamiento de $1 \mathrm{~km}$ y en área de amortiguamiento de $2 \mathrm{~km}$.

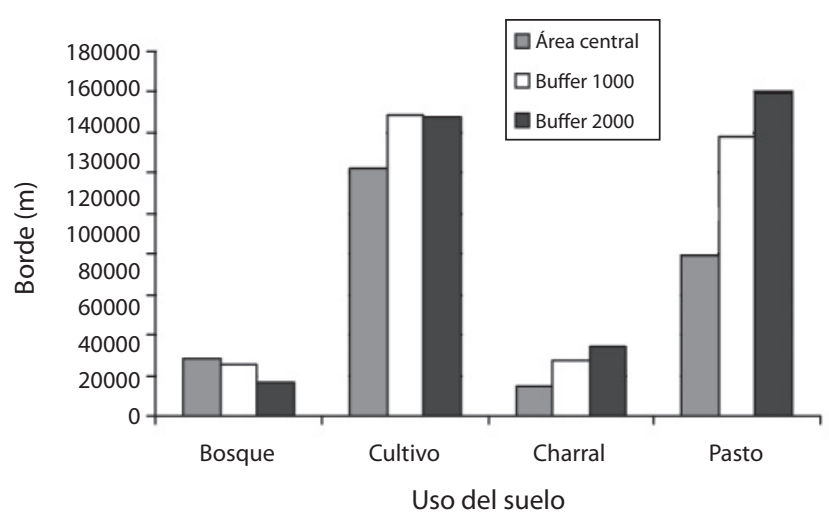

FIG. 7. Diferencia en borde total por clase de uso de suelo en la zona de estudio, en el área de amortiguamiento de $1 \mathrm{~km}$ y en el área de amortiguamiento de $2 \mathrm{~km}$. 
el efecto de borde ha crecido. La disminución del promedio del borde del parche en el bosque, el cultivo y el pasto en las zonas de amortiguamiento indica que los parches cada vez son más pequeños, lo cual es respaldado por el incremento en el número de parches y la disminución en el tamaño promedio de los mismos (Fig. 8). Lo anterior es indicativo de un incremento en la heterogeneidad del paisaje en una zona donde antes existía bosque, principalmente. Las implicaciones de esta situación sobre la vida silvestre son grandes; sin embargo, por la importancia de la zona como área de recarga de mantos acuíferos merece especial atención, ya que los parches de bosque, ideales para la recarga acuífera por la mayor capacidad de retención de agua, han disminuido y han sido remplazados por cultivos y pastos, los cuales tienen una menor capacidad de retención de agua. A futuro, esto podría significar escasez de agua en la Gran Área Metropolitana, la cual se abastece en un $80 \%$ de esta región (Ureña 2004).

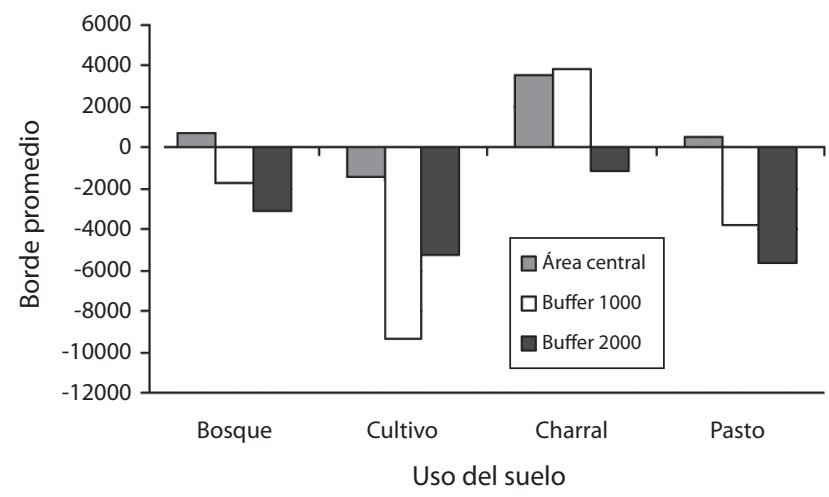

FIG. 8. Diferencia en el promedio del borde del parche por clase de uso de suelo en la zona de estudio, en el área de amortiguamiento de $1 \mathrm{~km}$ y en el área de amortiguamiento de $2 \mathrm{~km}$.

Según el estudio de Alvarado (2003), la tendencia en el cambio del uso del suelo indica que un $36 \%$ del bosque puede cambiar de uso para el año 2015, esto queda reforzado por las alteraciones al bosque que encontramos en este estudio. Generalmente, los otros usos son menos aptos para la captación de agua y el reabastecimiento de las aguas freáticas, por lo cual, si no se toman acciones activamente para manejar los bosques de esta zona, la recarga de este importante acuífero podría verse afectada en un futuro cercano.

\section{RECOMENDACIONES}

Los resultados obtenidos por medio del análisis de los mapas elaborados evidencian un proceso de cambio de uso del suelo. Los parches de bosque, ideales para la recarga acuífera por la mayor capacidad de retención de agua, han disminuido impactando negativamente en la captación de agua en la zona de recarga. Este hecho ha generado conflictos entre los diferentes actores sociales vinculados al área de estudio.

Dado que el área bajo estudio se caracteriza por la existencia de diferentes tipos de interés se recomienda iniciar un proceso de gestión participativa y de gobernabilidad sostenible del recurso hídrico en la zona de amortiguamiento del área protegida y así desarrollar acciones de manera conjunta con las comunidades ubicadas en la zona de influencia, con los gobiernos locales, asociaciones de desarrollo, instituciones públicas y privadas interesadas o directamente relacionadas con la protección, conservación y manejo sostenible de los recursos hídricos.

Para la elaboración de estrategias de conservación en la región, será necesario caracterizar y analizar en mayor profundidad el proceso actual de fragmentación al nivel de paisaje. Como también desarrollar investigaciones sobre el impacto de las prácticas de manejo de suelo en la zona de amortiguamiento del área protegida por el Decreto Ley LXV de 1888.

Se recomienda el desarrollo de inventarios de los cuerpos de agua utilizados y del caudal de explotación promedio, como de mapas de uso de suelo que permitan desarrollar un plan de manejo de la zona de recarga del Acuífero Barva, considerando la heterogeneidad (los diferentes paisaje, actores sociales y actividades económicas) que presenta la región.

Será necesario crear una reserva de protección absoluta dentro del área que protege el Decreto Ley LXV de 1888. Para ello se deberán establecer regulaciones y controles que contribuyan a disminuir la deforestación de los bosques primarios de las zonas de captación del recurso hídrico y regular el uso de la tierra en la región. Se recomienda iniciar un programa de reforestación por parte de la municipalidad o propietarios privados de las áreas de captación de los mantos acuíferos y los tributarios de esta región con plantas nativas, como también establecer un sistema de incentivos forestales para los propietarios de fincas privadas que deseen conservar la cobertura boscosa de sus propiedades.

Por último, como medida de mediano plazo se recomienda promover la adopción de una reglamentación especial para las áreas de captación de los mantos acuíferos que no están delimitadas por la Ley LXV del año 1888. 


\section{AGRADECIMIENTOS}

Agradecemos a J. F. Alfaro-Carvajal, de la Asociación Ambiental del Norte de San Rafael de Heredia y a Chris Vaughan, profesor asociado del Instituto Internacional en Conservación y Manejo de Vida Silvestre, la Universidad de Wisconsin-Madison y la Asociacion de Universidades del Medio Oeste, por el apoyo brindado durante la realización de la presente investigación.

\section{REFERENCIAS}

Alvarado, R. 2003. El plan regional metropolitano y los cantones de la GAM. Instituto de Fomento y Asesoría Municipal. Serie Cantones de Costa Rica, N. ${ }^{\circ} 4$. Costa Rica.

Baraona, M. \& G. Rivera. 1995. Desarrollo del jocote (Spondias purpurea L.) y del cas (Psidium friedrichsthalianum [Berg.] Niedz) en el bosque húmedo premontano de Costa Rica. Agronomía Mesoamericana 6: 23-31.

Bolaños, R. A. \& V. C. Watson. 1993. Mapa ecológico de Costa Rica según el sistema de clasificación de zonas de vida del mundo de Holdridge. Centro Científico Tropical. San José, Costa Rica.

Comisión Interinstitucional de Micro cuencas de Heredia (CIMH). 2005. Delimitación de las zonas de protección acuífera en las microcuencas de los ríos Ciruelas, Segundo, Bermúdez, Tibás y Pará. Programa CAMBIOS, EPPSUNA, San José, Costa Rica.

Environmental Systems Research Institute. 2002. ArcView GIS 3.3. Environmental Systems Research Institute, Inc. New Delhi, India.
Morera, C., M. Romero, P. Miranda, D. Avendaño \& M. Alfaro. 2007. Análisis del territorio periurbano, actividades ecoturísticas y paisaje de la Gran Área Metropolitana de Costa Rica. Informe final. Asistencia Técnica Local de Apoyo a la Gestión del PRUGRAM en la GAM. Universidad Nacional, Heredia, Costa Rica.

Ramírez, O. A. 2005. Estudio preliminar de la estructura del hábitat, configuración y composición del paisaje, basado en la distribución del quetzal (Pharomachrus mocinno costaricensis) en el volcán Barva, Costa Rica. Programa Regional en Manejo de Vida Silvestre para Mesoamérica y el Caribe Universidad Nacional, Heredia, Costa Rica.

Ramírez, R. \& A. Alfaro. 2002. Mapa de vulnerabilidad hidrogeológica de una parte del Valle Central de Costa Rica. Revista Geológica de América Central 27: 53-60.

Reynolds-Vargas, J. \& J. Fraile-Merino. 2002. Utilización de isótopos estables en la precipitación para determinar zonas de recarga del acuífero Barva, Costa Rica. IAEA-ECDOC-1611. Organismo Internacional de Energía Atómica, Viena, Austria.

Sistema Nacional de Áreas de Conservación (SINAC). 2007. Propuesta de ordenamiento territorial para la conservación de la biodiversidad de Costa Rica: Vol. 1. Análisis de vacíos en la representatividad e integridad de la biodiversidad terrestre Proyecto GRÚAS II, SINAC-MINAE. San José, Costa Rica.

Ureña, N. 2004. Efectos del aumento poblacional y del cambio de uso del suelo sobre los recursos hídricos en la Microcuenca del Río Ciruelas, Costa Rica. Tesis Mag. Sc. CATIE. Turrialba, Cartago, Costa Rica.

Vargas, A. 2002. Manantiales de una parte de Valle Central de Costa Rica. Revista Geológica de América Central 27: 39-52. 


\section{APÉNDICE 1}

Procesos legales y recursos de amparo que han sido aceptados por la Sala IV Constitucional con el fin de proteger la zona de recarga acuífera (José Francisco Alfaro-Carvajal com. pers. 2009):

N. ${ }^{\circ}$ de expediente y $\mathbf{N}^{\circ}$ de resolución: Exp: OS-014174-0007CO / Res. N² 2009012437.

Notificando: José Francisco Alfaro Carvajal (Asociación Ambiental del Norte de San Rafael de Heredia).

Motivo: Desplazamiento de tierra sobre el margen del río Turales por parte de la Municipalidad de San Rafael de Heredia.

N. ${ }^{\circ}$ de expediente y N. de resolución: Exp: 06-012316-0007CO / Res. № 2008004790.

Notificando: José Francisco Alfaro Carvajal.

Motivo: Señalan que en el distrito de Los Ángeles, San Rafael de Heredia se comenzó con el proyecto urbanístico "Brisas del Ciprés" el cual se ubica en una zona de recarga acuífera y de vulnerabilidad media a alta. Recurso de amparo contra la Municipalidad de San Rafael de Heredia y la Secretaría Técnica Nacional Ambiental.

N. de expediente y N. de resolución: Exp: 07-016118-0007CO / Res. № 2008010371.

Notificando: José Francisco Alfaro Carvajal.

Motivo: Se declaran contra la construcción del proyecto "Condominio Horizontal Residencial La Inmaculada", propiedad de Orlevi G S. A. ya que se encuentra en una zona de especial protección (recarga acuífera y media-alta vulnerabilidad). Recurso de amparo contra la Municipalidad de San Rafael de Heredia, Secretaría Técnica Nacional Ambiental (SETENA) e Instituto Nacional de Vivienda y Urbanismo (INVU).

N. ${ }^{\circ}$ de expediente y N. de resolución: Exp: 05-010758-0007CO / Res. № 2007003923.

Notificando: José Francisco Alfaro Carvajal y Ana Lorena Brenes Esquivel (Representación de Procuraduría General de la República).

Motivo: Solicita se declare la inconstitucionalidad de los Artículos 27 y 28 de La Ley Forestal que permiten la tala de árboles no nativos o autóctonos de la zona, sin importar el papel que éstos desempeñen en función de la protección de los recursos hídricos, el equilibrio ambiental y ecológico.

N. ${ }^{\circ}$ de expediente y $\mathbf{N} .^{\circ}$ de resolución: Sin información.

Notificando: Juan José Sobrado Ch.

Motivo: El Servicio Nacional de Aguas Subterráneas, Riego y Avenamiento (SENARA) actúa contra la vida y la salud de los habitantes de Costa Rica en el manejo de las aguas subterráneas para consumo humano. Demanda contra el SENARA.

N. ${ }^{\circ}$ de expediente y N. ${ }^{\circ}$ de resolución: Voto No. 2008-010371, de las 17 horas y 36 minutos del 19 de junio del 2008.

Notificando: José Ramírez Chávez (Asociación de Desarrollo Integral de Concepción de San Rafael de Heredia).

Motivo: Retrotraer los procedimientos y revisar nuevamente el proyecto Condominio Horizontal Residencial La Inmaculada a realizarse en Concepción de San Rafael. En este caso el recurso de amparo se hizo demandando a Municipalidad de S. Rafael, SETENA y Ministerio de salud.

N. de expediente y N. de resolución: Exp: 07-007996-0007CO / Res. No 2008-12109.

Notificando: Rafael A. Varela (Apoderado General de la Fundación para la Protección y Vigilancia de los Recursos Naturales de Heredia (PROCOVIRENA)).

Motivo: La Empresa de Servicios Públicos de Heredia S. A. está fomentando la contaminación y destrucción de las zonas de protección, al instalar redes eléctricas para la construcción privada de chalets, cabañas y hoteles de montaña en las zonas de recarga y reservorios de aguas subterráneas. Recurso de amparo contra: SENARA, Instituto Costarricense de Turismo (ICT), Ministerio de Salud, Ministerio de Agricultura y Ganadería (MAG), Ministerio del Ambiente, Energía y Telecomunicaciones (MINAET), Instituto Costarricense de Acueductos y Alcantarillados (ICCA), INVU, SETENA, Empresa de Servicios Públicos de Heredia (ESPH), municipalidades de Heredia, Santa Bárbara, Barva, San Pablo, San Isidro, San Rafael, Moravia y Vásquez de Coronado.

N. de expediente y N. de resolución: Exp: 98-004107-0007CO / Res: № 1998-06524.

Notificando: Carlos Guila Borrase (Apoderado General de CARGUILBO S. A.).

Motivo: Solicita se declare la inconstitucionalidad de los artículos 3 y 4 del Decreto Ejecutivo número 25902-MIVAH-MP-MINAE, ya que indican que los bienes inmuebles que se encuentran ubicados en la denominada "zona especial de protección", deberán tener un uso predominantemente agrícola y quedarán sometidos a diversas regulaciones sobre el tipo de edificaciones que se construyan en ellos. Alega que dichos artículos son contrarios al derecho de propiedad privada, mucho menos si no se brinda la indemnización correspondiente.

N. ${ }^{\circ}$ de expediente y N.º de resolución: Sin información.

Notificando: José Alberto Brenes André y Alvaro Sagot Rodríguez.

Motivo: Se declaran en contra de la adición de un inciso al Artículo 3 del Decreto Ejecutivo número 25.902-MIVAH-MP-MINAE, Plan Regional de Desarrollo Urbano del Gran Área Metropolitana, dado por Decreto Ejecutivo número 33757-MP-MIVAHMINAE, debido a que permite el desarrollo urbanístico en el anillo o límite de contención del Gran Área Metropolitana.

N. de expediente y N.º de resolución: Exp: AU-461-2004 / Res. No RRG-AU-14-2007.

Notificando: Judith Magnan Sins.

Motivo: Expone que la ESPH ofrece mal servicio de agua ya que es irregular. El recurso hídrico proveído está contaminado (sustancias orgánicas e inorgánicas indeseables), la cantidad de cloro que éste presenta es preocupante. Recurso de amparo contra la ESPH. 


\section{APÉNDICE 2}

De acuerdo con la información revisada, existe un gran número de instituciones y actores clave involucrados en la conservación del área, debido a que es una fuente importante de abastecimiento de agua para el Valle Central.

\begin{tabular}{|c|c|c|}
\hline Actores & Nombre de la organización & Acción \\
\hline \multirow[t]{3}{*}{ Sociedad civil } & Asociación Ambiental del Norte de San Rafael de Heredia & $\begin{array}{l}\text { Vigilancia } \\
\text { Protección y Control } \\
\text { Educación ambiental }\end{array}$ \\
\hline & $\begin{array}{l}\text { Movimiento regional por la defensa de las montañas del norte de Heredia, } \\
\text { de su ecosistema y su recurso hídrico }\end{array}$ & $\begin{array}{l}\text { Educación Ambiental } \\
\text { Protección y control }\end{array}$ \\
\hline & $\begin{array}{l}\text { Universidades: } \\
\text { Universidad Nacional (UNA) } \\
\text { Universidad de Costa Rica (UCR) } \\
\text { Universidad Estatal a Distancia (UNED) }\end{array}$ & Investigación \\
\hline \multirow[t]{6}{*}{ Gobierno } & Ministerio de Ambiente, Energía y Telecomunicaciones (MINAET) & Control y vigilancia \\
\hline & Dirección de Investigación del Parque Nacional Braulio Carrillo & Investigación \\
\hline & $\begin{array}{l}\text { Municipalidades (Barva, Heredia, San Rafael, Santa Bárbara, Federación de } \\
\text { Municipalidades de Heredia, Consejo de Desarrollo Regional de Heredia) }\end{array}$ & Control, protección y vigilancia \\
\hline & Servicio Nacional de Aguas Subterráneas, Riego y Avenamiento (SENARA) & Control \\
\hline & Empresa de Servicios Públicos de Heredia (ESPH) & Control y Educación Ambiental \\
\hline & Ministerio de Salud (MINSAL) & Control de calidad del agua \\
\hline Sector Privado & $\begin{array}{l}\text { Pequeños hoteles, ganaderos, beneficiarios por el Pago de Servicios } \\
\text { Ambientales (PSA) }\end{array}$ & $\begin{array}{l}\text { Producción agropecuaria y } \\
\text { servicios hoteleros }\end{array}$ \\
\hline
\end{tabular}

